\title{
Application of Local Polynomial Interpola- tion in Isomagnetic Map Magnetic Survey
}

\author{
Meng TianHua ${ }^{1,2}$ Du RuiQing ${ }^{1 *}$ Yang Jin $^{1}$ \\ ${ }^{1}$ School of Geophysics and Information Technology, China University of Geosciences \\ (Beijing), 100083, China \\ 2College of Physics and Electronics Science, Shanxi Datong University, Datong Shanxi \\ 037009, China
}

\begin{abstract}
The high density magnetic measurement data of one iron mine area was processed by local polynomial interpolation. The results indicate that contour map is high resolution and smooth when we choose polynomial mode 2 and search radius for $500 \mathrm{~m}$. That can provide the effective reference for the distribution of iron deposits. And it was feasible in analyzing magnetic data of mine using this method.
\end{abstract}

Keywords: interpolation algorithm; Parameter selection; search radius

\section{Introduction}

The characteristics of determination region are studied by isomagnetic maps during magnetic prospecting. Before drawing isomagnetic maps, we should deal with the data from txt to grid at first. The data gridding methods contain three types: distance weighting method, bearing point method and Surface spline interpolation grid method. The Surface spline interpolation grid method includes local polynomial interpolation, minimum curvature method and triangulation net/linear interpolation method and trend surface method. Local polynomial interpolation is a very commonly used nonparametric estimation method ${ }^{[1]}$. Kernel smoothing refers to a general class of techniques for non-parametric estimation of functions ${ }^{[2]}$. It can be useful in two classes of problems: density function estimation and non-parametric regression estimation. Local polynomial interpolation can overcome the defects of the general nonparametric kernel estimation in the boundary points of convergence speed which is lower than the within point. So it was considered to be an effective method for study the non-parameter model.

\section{Local Polynomial}

Local polynomial method, the ideas were originally presented by Stone CJ (1977). Be Clebeland (1979) and Katkovnik (1985) put the ideas applied in nonparametric regression model, Fan j. \& Gijbels i. (1996) made more detailed description for the local linear estimation ${ }^{[3-6]}$ In addition to the long-term trend, the variable quantities with short-range changes are important studied in earth science. When the data set shows short change, the local polynomial interpolation method can capture this change ${ }^{[7]}$. Local polynomial interpolation method is very sensitive to the neighborhood distance. The smaller search neighborhood may create an empty area in the forecast surface. Therefore, it is possible to preview the surface before output the layer.

Let $\mathrm{m}(\mathrm{x})$ is $\mathrm{p}+1$ order derivative,

$$
\beta_{j}\left(x_{0}\right)=m^{j}\left(x_{0}\right) / j !(\mathrm{j}=0,1,2, \ldots, \mathrm{p})
$$

Local polynomial methods select parame- 
ter $\beta_{j}\left(x_{0}\right)(j=0,1,2, \ldots, p)$, Show formula (1):

$$
\begin{aligned}
& \sum_{i=1}^{n}\left(Y_{i}-\sum_{j=0}^{p} \beta_{j}\left(x_{0}\right)\left(x_{i}-x_{0}\right)^{j}\right)^{2} \\
& K_{h}\left(x_{i}-x_{0}\right)=\min
\end{aligned}
$$

The formula (1) is written as formula (2):

$$
\begin{aligned}
& \sum_{i=1}^{n}\left(Y_{i}-\sum_{j=0}^{p} \beta_{j}\left(X_{i}-X_{0}\right)^{j}\right)^{2} \\
& K_{h}\left(x_{i}-x_{0}\right)=\min
\end{aligned}
$$

It is made

$$
Y_{t}=X_{t+d}
$$

The formula (3) is a kind of special case, indicating the $\mathrm{d}$ step prediction of the time sequence. When made the formula (3) of $d=1, p=0$, local polynomial fitting for degradation of kernel estimation as Nadaraya Watson estimator, see formula (4):

$$
f(x)=\frac{\sum_{t-1}^{T} Y_{t} K\left(\frac{X_{t}-x}{h}\right)}{\sum_{t=1}^{T} K\left(\frac{X_{t}-x}{h}\right)}
$$

Choosing Gauss kernel function when take advantage of local polynomial making forecast. Using the least squares fitting the sample data, the $\mathrm{T}$ moment of corresponding fitted value is actually the $\mathrm{X}_{\mathrm{T}+1}$ predicted value. This is because in the $\mathrm{T}$ point, the model is fitting used the value of before the point $T$, but does not use the sample information (the future information) of after $\mathrm{X}_{\mathrm{T}}$, so the fitting value is the predicted value (one-stepahead forecast), written as $\hat{X}_{T+1}$.

\section{Examples of Local polynomial me- thod in isomagnetic map magnetic survey}

\subsection{Data preparation}

A high-precision magnetic survey in one iron mine area has been carried out for one month in 2009. Thirty-two measuring lines are designed covering all the work area. The line numbers are planned at the beginning of 164 from west, and the maximum number is 216 in the east. The line numbers rise by 2 from west to east. The distance between the adjacent two measuring lines is 100 meters. There are about 235 measuring points in a single line. The point numbers are planned at the beginning of 180 , and they rise by 2 from south to north. The distance between the adjacent two measuring points in the same line is 20 meters.

Portable GPS(Global Positioning System) receivers are used to locate the measuring points, and proton magnetometers are used to measure the value of magnetic field intensity point by point. The data's units are nanotesla. Some of the data are shown in Tab.1.

Tab.1 cutting parameters of simulation milling

\begin{tabular}{cccccc}
\hline $\begin{array}{c}\text { Line } \\
\text { No. }\end{array}$ & $\begin{array}{c}\text { Point } \\
\text { No. }\end{array}$ & Data(nT) & $\begin{array}{c}\text { Line } \\
\text { No. }\end{array}$ & $\begin{array}{c}\text { Point } \\
\text { No. }\end{array}$ & Data(nT) \\
\hline 164 & 180 & 111.83 & 166 & 324 & -150 \\
\hline 164 & 182 & 105.77 & $\cdots$ & $\cdots$ & $\cdots$ \\
\hline 164 & 184 & 94.61 & 168 & 184 & 123.53 \\
\hline$\cdots$ & $\cdots$ & $\cdots$ & 168 & 186 & 124.3 \\
\hline 164 & 284 & -226.55 & 168 & 188 & \\
\hline 164 & 286 & -273.11 & $\cdots$ & $\cdots$ & $\cdots$ \\
\hline 164 & 288 & & 212 & 490 & -78.86 \\
\hline 164 & 290 & & 212 & 492 & -78.1 \\
\hline 164 & 292 & & $\cdots$ & $\cdots$ & $\cdots$ \\
\hline 164 & 294 & -326.08 & 214 & 550 & -20.04 \\
\hline 164 & 296 & -305.16 & 214 & 552 & -13.45 \\
\hline 164 & 298 & & $\cdots$ & $\cdots$ & $\cdots$ \\
\hline$\cdots$ & $\cdots$ & $\cdots$ & 216 & 550 & -24.72 \\
\hline 166 & 330 & -153.18 & 216 & 552 & -29.17 \\
\hline 166 & 322 & -151.45 & 216 & 554 & -28.81 \\
\hline & & & & &
\end{tabular}

Several lines' data are shown in Table 1, and several measuring points' data are 
missed because it is very difficult for workers to reach these points to measure. If we don't use natural neighbor method to interpolate data to fulfill the table, the isomagnetic map will have lots of empty regions. If that comes true, the result of calculating and the reference of magnet's location will be unreliable and with lowprecision.

\subsection{Contour interpolation algorithm}

It is very important to select appropriate polynomial model and the search radius for local polynomial interpolation. During drawing the contour map of this area, we chose variety of different polynomial model and search radius in order to find the best parameters. Through the comparative analysis, we get the better ultimately result is: choose the polynomial model 2 (M2) and the search radius for $500 \mathrm{~m}$ (R500) (Fig. 1 Fig. 4).

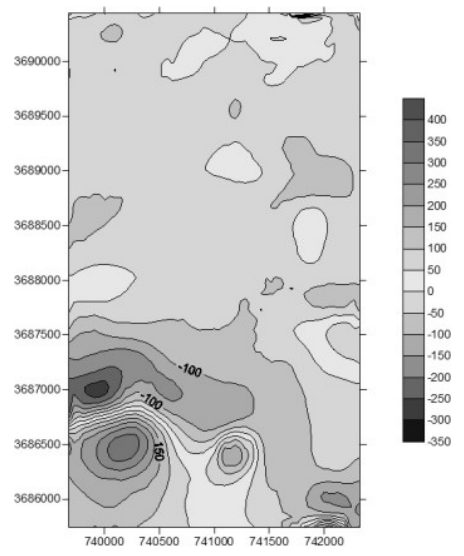

Fig.1: M1, R500

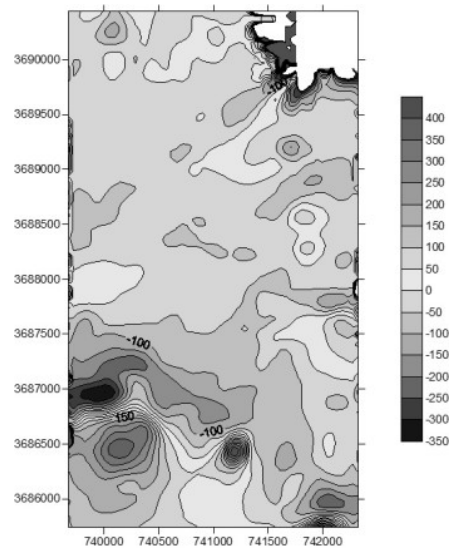

Fig.2: M2, R500

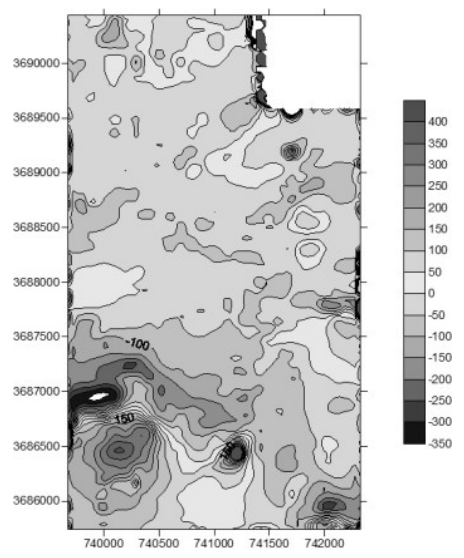

Fig.3: M2, R200

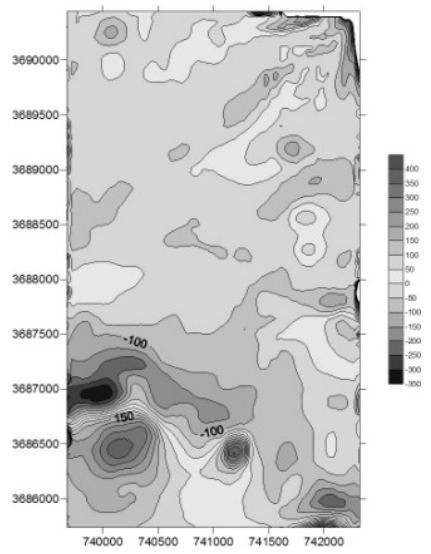

Fig.4: M2, R1000 
As can be seen from Figure 1 and Figure 2 , due to the correspond polynomial of mode 1 is simple, lowered the resolution, appeared invalid isogram in the blank area with no data, affected our correct judgment to the actual mineralize case of this region. It is obviously the more effective choice is mode 2 of local polynomial interpolation algorithm for analysis the contour maps in this region.

Compared Fig 2, Fig 3 and Fig 4, there are more details of the corresponding anomalies in the contour maps when the search radius is $200 \mathrm{~m}$. The actual distribution conditions of iron mine will be incorrect for these anomalies which confused the effective anomalies. When the search radius is 1000 , on the one hand, some valid anomaly details can be lost, on the other hand invalid data will be appeared for the search radius is too large in the blank area with no data, all of these result the negative impact to the metallogenic analysis. So the result of the search radius for $500 \mathrm{~m}$ is best, and a contour map with smooth and high-resolution would be gotten.

\section{Conclusion}

The results showed that the study of local polynomial interpolation is very effective to draw the contour maps. It can be analyze the distribution of ore deposits by choosing the right polynomial model and search radius. The theory of polynomial interpolation is simply and realized. It can eliminate the wave influence of moving revise and the effect of lower filter, maintain the real shape of amplitude and higher frequency information, and obtain the higher resolution contour map.

\section{References}

[1] Hardle W. Applied nonparametric regression [M]. Cambridge: Cambridge University Press. pp. 403-422, 1990.

[2] Wand M P. Jones M C Kernel Smoothing [M]. London: Chapman and Hall, pp. 348-368, 1995.

[3] Hart J D. Nonparametric smoothing and lack-of-Fit Tests Technometrics[J]. 41(2): 175-176, 1999.

[4] Michael G. Schimek, Estimation and inference in partially linear models with smoothing splines, Journal of Statistical Planning and Inference[J].91(2): 525-540, 2000.

[5] Hurvich C M. Simonoff J S.Tsai C I. Smoothing parameter selection in nonparametric regression using an improved A Kaike information criterion[J]. Journal of the Royal Statistical Society. Series B. 60:271-293, 1998.

[6] Mei C L. He S Y. Wang Y H. A note on the nonparametric least squares test for checking a polynomial relationship[J]. Acta Mathemation Applicate Sinica. 19:511-520, 2003.

[7] WANG Hui, CUI Ruo-Fei. Multiple Suppression Using T-A Dual Polynomial Fitting Method[M]. Journal of China University of Mining \& Technology, 9 (1): 55-59, 1999. 